\title{
ONLINE MONITORING OF REAL-TIME CARDIAC BEHAVIOR USING PORTABLE IoT TECHNOLOGIES
}

\section{JUAN PIEDRAHITA ${ }^{1}$, OCTAVIO JOSÉ SALCEDO PARRA², JUAN MANUEL SÁNCHEZ CÉSPEDES ${ }^{3}$}

${ }^{1}$ Ingeniero de sistemas. Integrante del grupo de investigación Internet Inteligente, Universidad Distrital Francisco José de Caldas, Facultad de Ingeniería. Bogotá D.C., Colombia

${ }^{2}$ Doctor en Ingeniería Informática, Doctor en Estudios Politicos, Magister en Teleinformática, Ingeniero de Sistemas, Profesor Titular y Director del grupo de investigación Internet Inteligente, Universidad Distrital Francisco José de Caldas, Facultad de Ingeniería. Bogotá D.C., Colombia

${ }^{3}$ Magíster en Administración, Especialista en Teleinformática, Ingeniero Electrónico. Profesor Asociado e integrante de los grupos de investigación GIIRA y AQUAFORMAT, Universidad Distrital Francisco José de Caldas, Facultad de Ingeniería. Bogotá D.C., Colombia

ABSTRACT
Heart diseases are the worldwide leading cause of death, even though they can be prevented through continuous
monitoring of the patient. This paper presents the development of a device that uses different sensors to recollect
information on the behavior of the heart (Rhythm, electric activity and blood pressure). IoT technologies are used to
store said information into a database and show it in real time which enables continuous monitoring of the patient
with $99.93 \%$ reliability. Furthermore, in case of detecting abnormal behavior, the device sends a notification to a
relative, specialist or guardian chosen by the patient.
KEYWORDS: Cardiac disease, Continuous Monitoring, Individual medical control \& IoT

Received: Nov 30, 2020; Accepted: Dec 21, 2020; Published: Dec 24, 2020; Paper Id.: IJMPERDDEC202060

\section{INTRODUCTION}

The concept of telemedicine or mobile medical healthcare is becoming more relevant since the idea of receiving inhome care is more attractive to people than leaving their homes for check-ups or going to appointments. Advances in information and communication technologies enable to technically monitor the parameters related to the health of patients wherever the user may be [1].

Among the most common systems of individual health monitoring, the monitoring systems used for glucose, cardiac rhythm and electric cardiac activity (electrocardiogram) [2]. The most used systems are those that track cardiac activity since the leading cause of death all over the world are heart diseases. Initially, the idea of monitoring the heart only implied tests carried out in hospitals with cutting-edge technology. Nonetheless, over time portable sensors became more accessible to people in devices that could be purchased for individual monitoring at home.

Portable sensors offer different advantages including the ability to be carried anywhere as well as data management

functions such as recollection, processing, momentary storage and transfer to devices connected to the internet (mobile devices or remote servers) [3]. Although this technology is currently available, it is not enough since most users do not know how to properly read or interpret the data and determine whether the results are good or bad 
news. Furthermore, the use of devices is restricted to short time periods since the battery is insufficient and the sensors must provide accurate biomedical data due to the importance of the patient's information [4]. Low consumption technologies must be used that can operate during long time lapses.

On another matter, applications do not have persistence in data meaning that the recollected data do not represent the information of patients over long periods of time but are rather restricted to one time session or only reunite the main characteristics or relevant data which makes information unavailable for possible future inquiries. Seeking to solve the previously discussed issues, it is proposed to develop and implement a system that allows users to monitor cardiac behavior by measuring the heartbeat, the electric activity of the heart and the blood pressure. The recollected values are stored so they can be browsed at any moment, even in real time, so that both patients and authorized people (relatives or specialized professionals) can have access to the information. The server itself can generate notifications or inform someone in case of abnormal behavior.

\section{BACKGROUND}

There are various researches dwelling on individual and remote monitoring of patients, focused on supervising vital signals through the use of technologies. Nowadays, a wide variety of sensors are available on the market that can monitor any type of vital sign. They can be mounted over a hardware layer and show data to users or send it to other devices such as cellphones and display it using an application or on remote servers to be downloaded from any place with access to internet.

Sensors can be placed in different devices. For instance, a strip is designed for the elderly in [5] that can measure the heartbeat, detect falls and emit notifications when an abnormal conduct is presented. In this case, the strip is placed on the wrist. It can also be placed on the chest [6] for athletes and measure the cardiac activity during intense physical workouts with the help of the segMA algorithm. The interference of the ECG signal is suppressed which delivers more accurate measurements. Some examples can be seen in [7][8], where the operation of the device is explained and the authors converge on the solution based on portable monitoring gadgets stating that they require diodes to be connected to specific parts of the body which makes their use quite difficult. This approach can be discarded if a heartbeat monitor is used that includes embedded sensors and is placed on a specific location.

Another approach consists on cellphone applications which can be divided into two variations:

- Applications that use the sensors of the device to gather data such as using the cellphone's flashlight as a LED sensor and apply photoplethysmography to read vital signs such as heartbeat [9][10] or blood pressure [11].

- Applications that can be connected to external sensors and manipulate the collected data as in [12] where a lowcost device was conceived as a cellphone case and contained ECG and heartbeat sensors that also connects to an application installed in the cellphone to send and show information.

The problem with the second option is that the portability of the device restricts the monitoring to short sessions since the user cannot carry the device at all times for check-up. Other examples can be found in [13] [14], where low consumption technologies are used such as Bluetooth Low Energy (BLE) and SMS or online notifications.

Other approaches proposed to place sensors are not very common but they are described in literature such as [15], where a monitor for ECG and blood pressure is shown in which the sensors are placed on jacket or shirt, granting access to the internet to send data to nearby devices. Another case is discussed in [16] where the sensors are placed on a chair and 
perform a diagnosis of the patient in approximately 7 minutes. This chair is designed to be available on pharmacies and reduce the gap between medical services and rural populations where they are limited. As seen in other articles, there is an issue with constant portability and monitoring in this scenario.

Some of the current problems of telemedicine are related with continuous monitoring and the fact that storing the information collected by the sensors requires a high storage capacity. For instance, if an application stores the heartbeat values of a patient each second, it needs to store 86400 records every day. Another example is shown in [17] where an estimation is carried out on the bytes required to store the medical information of all people living in India.

In addition to the storage problem, the security and privacy of the data is another matter [18] since the patient can decide with whom his information is shared being a delicate subject for some people. These aspects must be considered during the development of the app to avoid future problems.

The previously described work shows that the technologies developed until now have had problems with guaranteeing a continuous monitoring in terms of energy consumption since the sensor cannot be used constantly. Another problem concerns the sensor location of the sensor which can be misplaced by the user and thus affect the accuracy of the measurements thereby deliver incorrect results. These issues can be solved with the use of Bluetooth Low Energy (BLE) and sensors placed in one single location easily identified by users. Finally, regarding the security issue, login can be required for the application so that only the user and people authorized by the user can view the medical data.

\section{METHODOLOGY}

To carry out the development of the continuous monitoring system, it is necessary to define the sensors that will be used so that they can connect through BLE with the application using the Bluetooth protocol already included in the user cellphone. The sensor chosen is the heartbeat sensor HR-12 from CATEYE which is coupled to the previously described conditions and does not require any additional accessories to manage the connection. This sensor is connected to the chest of the user at the same height as the heart and comfortably adjusts to the body so it can be used at any time. A complete specification of the device characteristics is shown in [19].

A database model was developed that allows the management of the persistence of information for each user of the device. The database is relational and developed with the PostgreSQL engine. It stores the basic information of each patient registered in the system, the measurements carried out by the sensors and the contact information of the people that patients wish to notify in case of an emergency.

To manage the database information and each operation, an API is used with REST services that can be consumed through HTTP requests from any web client or even from the app. This server notifies the users defined by the patient in case of abnormal behavior.

Once the device, the persistence and the information management are defined, an app is created with the Android studio tool that works as a bridge between the data collected by the sensors and their persistence, given that the data is sent from the sensors to the REST API in order to be stored and/or consulted. Additionally, the app has different notification means in case of detecting irregular behavior in the data.

Thus, the mechanisms required to carry out the tests with different people are defined as well as an assessment of the obtained results and statements regarding future work on research. 


\section{DESIGN}

The architecture of the system to be developed. Each component of the system is detailed and how they communicate between them. Their characteristics and functions give support to the communication and are hereby listed:

Heartbeat monitor. The heartbeat sensor must operate under the BLE protocol. The sensor exposes services which are consumed by other devices that operate under this communication protocol. The services are called GATT (Generic Attribute Profile) which establish in detail how the exchange of information is carried out through the BLE connection. Each service has a unique identifier that describes its function and sets it apart from the others. The identifiers are standardized in the GATT service specification in [20]. The chosen sensor can be seen in Fig. 1.

REST API. This component is in charge of all the operations of a CRUD, i.e., it allows to create, read, update and erase information in the database. Additionally, it contains the procedures that identify when is it necessary to notify a patient when abnormal behavior is detected in the data.

DB Server. The database is in charge of keeping all the basic information of the user and the collected measurements. The information can only be accessed from the REST API and seen from the application or the web client. The database model exhibiting the tables required to register the information of the patients, their contact information, the people to be notified and each measurement recorded by the sensor.

Android device with continuous monitoring application. An application is developed for Android platforms that must be compatible with the BLE technology, since the application uses the Bluetooth connection of the cellphone to connect to the services exposed by the sensor. When a connection is established with the heartbeat monitor and the measurements are sent and read by the app, they are shown to the user and sent to the server to be stored. The application sends a new registry of the measurement to the server for every second of monitoring.

Web client. The development of the web client uses the Angular framework and consumes the REST API services through HTTP requests in order to show the information to users that are browsing. It is noteworthy to mention that both the web client and the Android application must implement a login command that identifies the person and the role it has in the system so that the permissions are clearly established.

\section{IMPLEMENTATION}

The first part of the implementation consisted on developing the server (see code in [21]), which uses the postgresDB library [22] to connect with the database and the Express library [23] to expose the following endpoints of the server:

- Login: It allows the user to login with his identification and password.

- Get Measurements: It obtains the measurements of heartbeat for all patients. If an identifying parameter is passed, the result only contains the measurements related with said identifier.

- Post Measurement: It stores the measurements of the user into the database.

- Get Patients: It obtains the data of the patient. If an identifying parameter is passed, the result only contains the measurements related with said identifier.

- Post Patient: It registers a patient and stores his basic information.

- Get Diagnosis: It reads the diagnosis linked to the patient that is currently browsing.

- Post Diagnosis: It stores the results of the diagnosis when the user chooses this option in the application. 


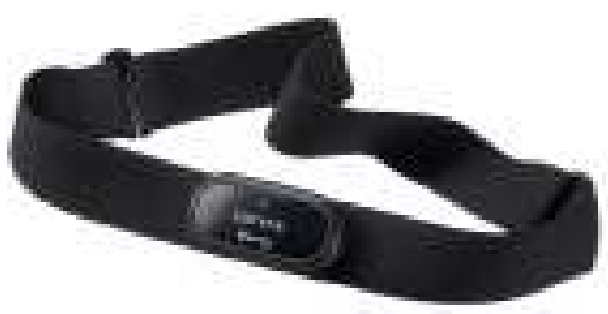

Figure 1: HR-12 Heartbeat sensor from CATEYE. Source: CATEYE Co.

In addition to the server, an Android application was developed that can connect with the CatEye sensor through BLE. Therefore, the cellphone must support Bluetooth 4.0 or higher. The application starts by asking the login information of the user. Once the login is confirmed, the initial screen is shown (Fig. 2) where not connection has been established with the sensor. Once the BLE sensor is chosen, the measurements of the heartbeat are displayed to the user while being sent to the server for storage.

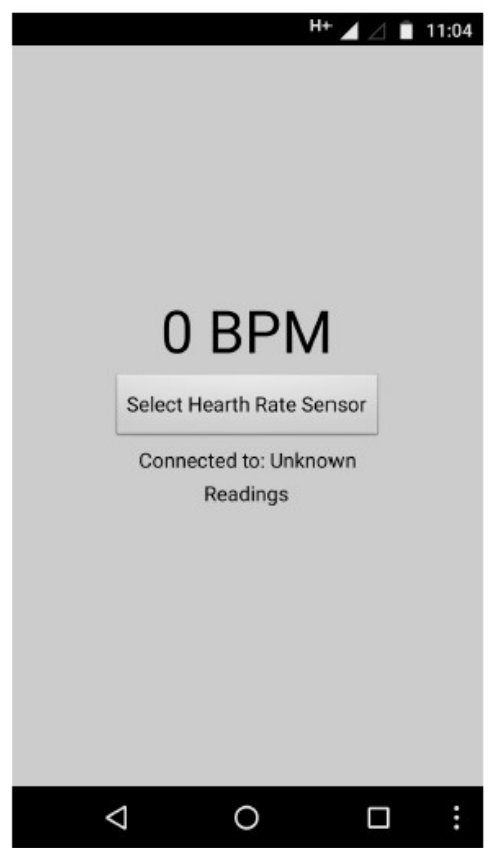

Figure 2: Start of the application before establishing connection with the BLE sensor. Source: Authors. 


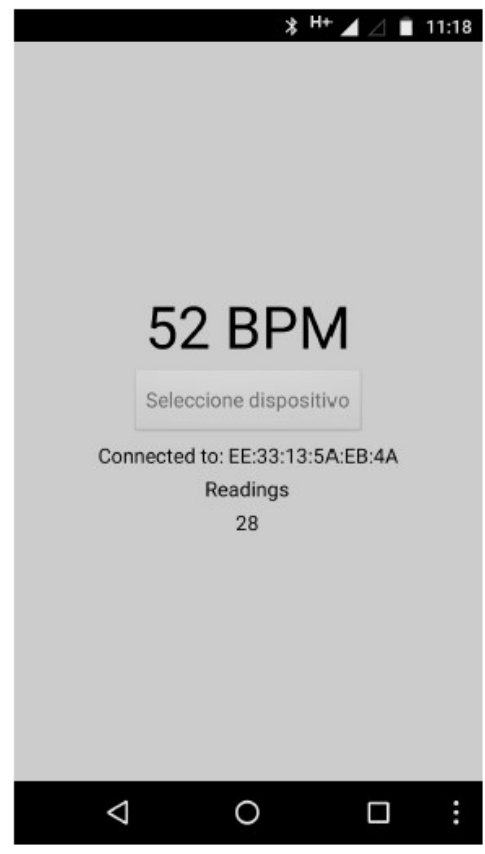

Figure 3: Start of the application after establishing connection with the BLE sensor and first readings. Source:

\section{Author}

In addition to measurements, in case the application reads a very high or very low value (over 140 or below 40 ) it will send an alert to a contact chosen by the user with the current location of the patient. In order to assure that the contacted person receives the message in a timely manner and can respond to the emergency, the message is sent through different channels:

- SMS: The cellphone connection is used and the SMS to send a message to the number of the chosen contact.

- $\quad$ E-mail: The server sends an e-mail to the chosen contact.

- Telegram: With the help of instant messaging apps, the Telegram app sends a message to the cellphone number of the chosen contact.

\section{DISCUSSIONS}

As a result, a system was designed with the proposed characteristics. The different parts of the system are:

- Database: Developed in PostgresDB, it enables the persistence of data for users of the system.

- API REST: It is an API that allows users of the mobile application to access the available data of the database after a simple login.

- Mobile application: Developed for Android devices, the application allows the user to connect to different BLE sensors to monitor vital signs permanently and keep a record of the measurements obtained.

- BLE sensor of vital signs: The sensors with BLE technology connect with the mobile application through generic addresses of the UUID services and monitor the vital signs of the patient in real time. 
This system includes the following characteristics that give it some advantages compared to other alternatives and studies proposed:

Table 1: Comparative table of sensors

\begin{tabular}{|c|c|c|c|}
\hline Proposal & $\begin{array}{c}\text { Dimensions } \\
(\mathbf{c m})\end{array}$ & $\begin{array}{c}\text { Battery } \\
\text { (Type, Duration) }\end{array}$ & Device location \\
\hline $\begin{array}{c}\text { Proposed by authors CatEye } \\
\text { HR-11 sensor }\end{array}$ & $1 * 1 * 2$ & BLE, 1 year & Chest band \\
\hline$[12]$ & $3 \times 2 \times 3$ & AAA, 1 week & Chest nodes \\
\hline$[16]$ & Office chair & Depends on electric current & Home \\
\hline
\end{tabular}

- Easy to use: In addition to an intuitive application, the BLE sensors are very easy to link and use. It is recommended to place the sensors in the chest since they are more comfortable according to [8], being preferred by 8 out of 10 patients, even while sleeping. Furthermore, the use of sensors instead of modules allows users to use the system during the entire day. Table 1 compares the proposed sensor with complete modules discussed in some referenced articles.

- Reliability of the sensors: Table 2 shows the comparison of the reliability of the sensors [13] and the results obtained with the CatEye HR-11 sensor [19].

- Autonomy: BLE sensors have a battery that can last up to a year which leaves behind the need to connect the device for recharge and use. The solutions proposed in [5] do not offer this degree of autonomy for the user.

Table 2: Comparative table of sensor reliability

\begin{tabular}{|c|c|c|c|c|c|c|}
\hline \multirow[t]{2}{*}{ Sensor } & \multirow[t]{2}{*}{$\mathbf{N}$} & \multicolumn{2}{|c|}{ Measurement } & \multirow{2}{*}{$\begin{array}{l}\text { Error } \\
(\%)\end{array}$} & \multirow[t]{2}{*}{ Total } & \multirow[t]{2}{*}{ Reliability } \\
\hline & & Test 1 & Test 2 & & & \\
\hline \multirow{10}{*}{ [13] } & 1 & 73 & 75 & -2.74 & \multirow{10}{*}{1.66} & \multirow{10}{*}{$98.34 \%$} \\
\hline & 2 & 71 & 70 & 1.43 & & \\
\hline & 3 & 93 & 92 & 1.43 & & \\
\hline & 4 & 59 & 60 & -1.43 & & \\
\hline & 5 & 69 & 69 & 0 & & \\
\hline & 6 & 72 & 73 & -1.43 & & \\
\hline & 7 & 71 & 75 & -5.63 & & \\
\hline & 8 & 65 & 71 & -9.84 & & \\
\hline & 9 & 75 & 77 & -2.67 & & \\
\hline & 10 & 96 & 92 & 4.17 & & \\
\hline \multirow{10}{*}{ Proposal } & 1 & 75 & 73 & 2.67 & \multirow{10}{*}{0.07} & \multirow{10}{*}{$99.93 \%$} \\
\hline & 2 & 70 & 71 & -1.43 & & \\
\hline & 3 & 75 & 76 & -1.43 & & \\
\hline & 4 & 59 & 59 & 0 & & \\
\hline & 5 & 70 & 69 & 1.43 & & \\
\hline & 6 & 72 & 73 & -1.43 & & \\
\hline & 7 & 71 & 75 & -5.63 & & \\
\hline & 8 & 70 & 65 & 7.14 & & \\
\hline & 9 & 77 & 75 & 2.67 & & \\
\hline & 10 & 92 & 95 & -3.26 & & \\
\hline
\end{tabular}


Data persistence: In contrast with the proposals in [14] that cannot store the data gathered by users, this system periodically stores every measurement recorded by the sensors connected to the server. The measurements are associated with the user and the time that they were sent. Depending on the connected sensor, there is a different time period to send data. The interval for each sensor can be seen in Table III.

Table 3: Comparative table of time intervals to send data from the application to the server

\begin{tabular}{|c|c|}
\hline Sensor & Interval (seconds) \\
\hline Heartbeat & 1 \\
\hline Blood pressure & 60 \\
\hline Arterial pressure & 600 \\
\hline
\end{tabular}

- Security: According to [3], one of the weakest aspects of the systems for permanent monitoring of vital signs is security since hackers can access the data of the patients by re-engineering the mobile applications as proposed in [5] and [13]. This allows the patients to access the data since the application does not carry out any persistency for the user information and assures through authentication that only the user can have access to said information.

- Alert: This system seeks to guarantee the opportune delivery of the notification through the use of different channels including SMS applications as seen in [13] [14]. SMS messages are subjected to the availability of the mobile service of the user and the contact receiving the alert.

\section{CONCLUSIONS}

Both the developed device and the conceived design meet the objectives for which they were proposed. An IoT system was implemented for the continuous monitoring of the vital signs (blood pressure, arterial pressure, heartbeat) with a persistency in the measurements and immediate notifications in case of abnormal behavior in the sensor measurements.

Storing the data in a server that can only be accessed through authentication instead of a local app adds a security component that allows the information of the users to only be accessed by them.

BLE sensors offer a high reliability rate $(99.93 \%)$ which confirms that they are a good choice for the field of telemedicine.

The location of the sensors makes it easy to use the device which is also comfortable to wear even while sleeping.

The use of BLE technologies significantly increases the autonomy of the device since the user does not depend on an outlet to use or recharge its batteries. The device can be used for over a year with no need to worry about the battery.

Using various channels to send the alert message guarantees their timely delivery and the swift response of the contacted person. This is an advantage of the system compared to other alternatives that are restrained by the user's telephone service regarding the available SMS.

As stated in [16], there is a social resistance against changing how vital signs are monitored. It is paramount that these barriers are suppressed keeping in mind that current technologies are highly accurate. 


\section{REFERENCES}

1. A. Bhatti, A. A. Siyal, A. Mehdi, H. Shah, H. Kumar, and M. A. Bo- hyo, "Development of cost-effective tele-monitoring system for remote area patients," in 2018 International Conference on Engineering and Emerging Technologies (ICEET), Feb 2018, pp. $1-7$.

2. N. Kumar, "Iot architecture and system design for healthcare systems," in 2017 International Conference On Smart Technologies For Smart Nation (SmartTechCon), Aug 2017, pp. 1118-1123.

3. F. Nausheen and S. H. Begum, "Healthcare iot: Benefits, vulnerabilities and solutions," in 2018 2nd International Conference on Inventive Systems and Control (ICISC), Jan 2018, pp. 517-522.

4. H. Alshaheen and H. Takruri-Rizk, "Energy saving and reliability for wireless body sensor networks (wbsn)," IEEE Access, vol. 6, pp. $16678-16695,2018$.

5. T. Elakkiya, "Wearable safety wristband device for elderly health monitoring with fall detect and heart attack alarm," in 2017 Third Inter-national Conference on Science Technology Engineering Management (ICONSTEM), March 2017, pp. $1018-1022$.

6. S. M. A. Salehizadeh, Y. Noh, and K. H. Chon, "Heart rate monitoring during intense physical activities using a motion artifact corrupted signal reconstruction algorithm in wearable electrocardiogram sensor, " in 2016 IEEE First International Conference on Connected Health: Applications, Systems and Engineering Technologies (CHASE), June 2016, pp. 157- 162.

7. A. K. Ahmadi, P. Moradi, M. Malihi, S. Karimi, and M. B. Shamsollahi, "Heart rate monitoring during physical exercise using wrist-type pho- toplethysmographic (ppg) signals, " in 2015 37th Annual International Conference of the IEEE Engineering in Medicine and Biology Society (EMBC), Aug 2015, pp. 6166-6169.

8. M. Simonnet, B. Gourvennec, and R. Billot, "Connected heart rate sensors to monitor sleep quality: Electrodes, chest belt and smartwatch users acceptability, " in 2016 IEEE First International Conference on Connected Health: Applications, Systems and Engineering Technologies (CHASE), June 2016, pp. 344-345.

9. M. Ghamari, C. Aguilar, C. Soltanpur, and H. Nazeran, "Rapid prototyp-ing of a smart device-based wireless reflectance photoplethysmograph," in 2016 32nd Southern Biomedical Engineering Conference (SBEC), March 2016, pp. 175-176.

10. S. Sukaphat, S. Nanthachaiporn, K. Upphaccha, and P. Tantipatrakul, "Heart rate measurement on android platform," in 2016 13th Inter- national Conference on Electrical Engineering/Electronics, Computer, Telecommunications and Information Technology (ECTI-CON), June 2016, pp. 1-5.

11. R. Nye, Z. Zhang, and Q. Fang, "Continuous non-invasive blood pressure monitoring using photoplethysmography: A review," in 2015 International Symposium on Bioelectronics and Bioinformatics (ISBB), Oct 2015, pp. 176-179.

12. M. S. Mahmud, H. Wang, A. M. Esfar-E-Alam, and H. Fang, "A wireless health monitoring system using mobile phone accessories, " IEEE Internet of Things Journal, vol. 4, no. 6, pp. 2009-2018, Dec 2017.

13. A. L. Valor, M. R. B. Apsay, J. R. M. Acebo, A. Aguilar, C. J. B. Onquit, and M. G. Chua, "Heartsaver: A heart rate monitoring system with sms notification, " in 2016 IEEE Conference on Systems, Process and Control (ICSPC), Dec 2016, pp. 1-6.

14. M. F. B. Mustapha and T. Anwar, "Mobile heart rate monitor for myocardial infarction patients," in 2017 6th ICT International Student Project Conference (ICT-ISPC), May 2017, pp. 1-4.

15. D. M. D. Ribeiro, M. F. M. Colunas, F. A. F. Marques, J. M. Fernandes, and J. P. S. Cunha, "A real time, wearable ecg and continous blood pressure monitoring system for first responders," in 2011 Annual Inter- national Conference of the IEEE Engineering in Medicine and Biology Society, Aug 2011, pp. 6894-6898. 
16. S. Sarkar and R. Saha, "A futuristic iot based approach for providing healthcare support through e-diagnostic system in india," in 2017 Second International Conference on Electrical, Computer and Communication Technologies (ICECCT), Feb 2017, pp. $1-7$.

17. P. Grover and R. Johari, "Review of big data tools for healthcare system with case study on patient database storage methodology," in 2016 6th International Conference - Cloud System and Big Data Engineering (Confluence), Jan 2016, pp. 698-700.

18. M. Bahrami and M. Singhal, "A dynamic cloud computing platform for ehealth systems, " in 2015 17th International Conference on E-health Networking, Application Services (HealthCom), Oct 2015, pp. 435-438.

19. C. CO. Cateye heart rate sensor hr-11 especifications. [Online].Available: https://www.cateye.com/manual/en/sensor portal/guide2 spec.html\#hr- 11

20. B. SIG. Gatt services. [Online]. Available: https://www.bluetooth.com/specifications/gatt/services

21. J. Piedrahita. Servidor de aplicacin de ritmo cardiaco en node.js. [Online]. Available: https://github.com/JuanPiedrahita/ritmoCardiaco

22. B. C. node-postgres. [Online]. Available: https://github.com/brianc/node-postgres

23. N. Foundation. Express. [Online]. Available: https://expressjs.com/ 